
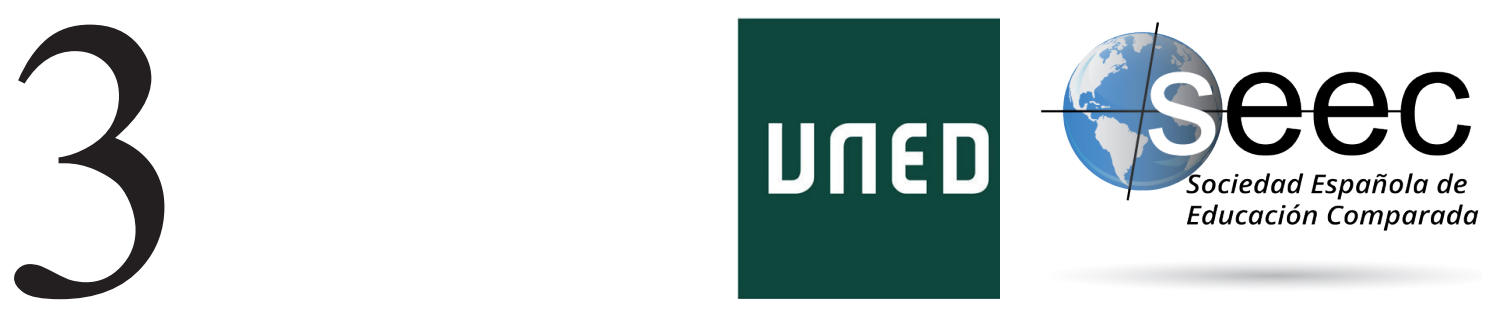

\title{
La cooperación interuniversitaria Canarias-Cabo Verde: perspectivas complementarias, perspectivas enfrentadas
}

\author{
The interuniversity cooperation between \\ Canary Islands and Cape Verde: complementary \\ perspectives, confronting perspectives
}

\section{Chamaida Inés Tabares Márquez*; María Inmaculada González Pérez**}

DOI: $10.5944 /$ reec.40.2022.30311

\author{
Recibido: 18 de marzo de 2021 \\ Aceptado: 10 de septiembre de 2021
}

\begin{abstract}
*Chamaida Inés Tabares Márquez: Licenciada en Pedagogía y Diplomada en Estudios Avanzados en Sociología dentro del programa de Doctorado «Sociedad, Política y Cultura» por la Universidad de La Laguna. Postgraduada en Relaciones Internacionales por la Universidad de Glasgow y en Gerencia Internacional y Liderazgo por la Universidad Queen Margaret en Edimburgo. Actualmente desarrolla su tesis doctoral en Educación por la Universidad de La Laguna y ha realizado diversas instancias de investigación internacionales para su logro en la Universidad de Cabo Verde y en la Universidad de Glasgow. Datos de contacto: E-mail:aluo100098549@ull.edu.es

**María InMaculada González Pérez: Doctora en Pedagogía y profesora de Educación Comparada y Educación para la Cooperación y el Desarrollo en la Universidad de La Laguna. Su campo de investigación se ha centrado en la cooperación educativa internacional y en el reconocimiento de la diversidad cultural en el marco de los Derechos Humanos. Anteriormente a las funciones de profesora trabajó para la Organización Internacional del Trabajo en Bolivia, Costa Rica, Nicaragua, Panamá, El Salvador y Guatemala y para la Agencia Española de Cooperación Internacional en Costa Rica. Desde el ámbito universitario ha dirigido varios proyectos en colaboración con Cabo Verde, Mali, Mauritania y Marruecos. Datos de contacto: E-mail: migonpe@ull.edu.es
\end{abstract}




\title{
Resumen
}

Con el fin de que la cooperación interuniversitaria contribuya al desarrollo del mundo y particularmente de África y Canarias, este trabajo profundiza en la visión de los protagonistas de los procesos de cooperación entre la Universidad de La Laguna (ULL) (España) y la Universidad de Cabo Verde (UniCV) (Cabo Verde) desde 2015 hasta 2019. Para ello, se contó con la colaboración de informantes clave de ambas universidades: cargos de responsabilidad, docentes y personal de administración. Los resultados presentados se alcanzaron por medio de un estudio comparativo de carácter cualitativo sobre sus concepciones de la cooperación internacional y la cooperación interuniversitaria al desarrollo. La visión de estos agentes respecto a la puesta en prácticas de estos conceptos claves, también fue objeto de análisis. Se hallaron perspectivas a veces enfrentadas, a veces complementarias, en función de todas las variables investigadas; especialmente en lo que respecta a la vinculación existente entre las personas entrevistadas y cada una de las universidades. Esta es la razón que ha motivado el presente trabajo. Sus propuestas de mejora de la cooperación interuniversitaria al desarrollo han sido especialmente valoradas bajo una visión prospectiva, tratando de ofrecer novedosas posibilidades a las universidades comprometidas con un futuro común del planeta..

Palabras clave: Cooperación Internacional; Cooperación Interuniversitaria; Desarrollo; Canarias; Cabo Verde.

\begin{abstract}
In order for interuniversity cooperation to contribute to the development of the world and particularly of Africa and the Canary Islands, this work deepens into the vision of the protagonists of the cooperation processes between the University of La Laguna (ULL) (Spain) and the University of Cape Verde (UniCV) (Cape Verde) from 2015 to 2019. For this, we had the collaboration of key informants from both universities. Who do hold positions of responsibility, as Lecturers, management...The results presented were achieved through a qualitative comparative study on their conceptions of international cooperation and interuniversity cooperation for development. The vision of these agents regarding the implementation of these key concepts, was also analysed. Sometimes conflicting, sometimes complementary perspectives were found in relation to all the variables taken into consideration. Particularly in relation to the position of responsibility of the people who were interviewed and the Universities to where they belong to. Their proposals for improving interuniversity cooperation for development have been especially valued under a prospective vision. As they are trying to offer new possibilities to universities. Which, furthermore, are committed to a common future of the planet.
\end{abstract}

Keywords: International Cooperation; Interuniversity Cooperation; Development; Canary Islands; Cape Verde. 


\section{Introducción : Cooperación Interuniversita- ria al Desarrollo}

La cooperación interuniversitaria al desarrollo constituye un ámbito específico de la cooperación educativa internacional, la cual persigue trabajar desde la óptica del compromiso con unas relaciones Norte-Sur más equitativas, justas, solidarias y de emancipación del Sur proponiendo contenidos, conceptos, procedimientos y actitudes que también permiten un análisis de los problemas de manera crítica sobre el origen de los mismos, su comprensión y su solución desde el respeto a los Derechos Humanos (García de la Torre, 2013, p. 67).

Esta debe entenderse como un trabajo conjunto entre el Norte y el Sur que contribuya a la búsqueda de soluciones globales para los problemas globales que hoy afectan al mundo (Kabunda, 2011).

La cooperación educativa internacional ha sufrido un proceso de evolución y ya en el siglo XXI, se encuentra impregnada del concepto de Desarrollo Humano Sostenible, atendiendo a los siguientes principios: erradicar la pobreza, promover la diversidad cultural, promover la igualdad de género, extender la enseñanza primaria universal, así como la sostenibilidad y la participación (González y García de la Torre, 2006). En base a este concepto, la cooperación educativa internacional se concibe como:

Para fomentar el desarrollo sostenible y mejorar el bienestar social. Y ello requiere sobre todo del derecho a la educación y ello implica, entre otros factores, la disponibilidad de profesionales altamente cualificados y la capacidad de la sociedad para innovar y adaptar las tecnologías y los conocimientos a un contexto nacional específico. Las universidades públicas ofrecen las habilidades, conocimientos y actitudes que contribuyen al desarrollo de sus países por medio de sus cuatro ámbitos principales: la formación y el desarrollo del capital humano; la investigación científica; el desarrollo social y cultural; la innovación y el progreso económico.

La propia definición de las universidades públicas como espacios de generación y transmisión de conocimientos no sabe de fronteras, por lo que se mide en un contexto mundial globalizado, donde estas, desde hace muchos años han desarrollado actividades diversas que contribuyen al desarrollo internacional: desde proyectos colaborativos de investigación, programas de formación conjuntos, intercambios de estudiantes y profesorado, hasta actividades de transferencia de conocimiento (Vilalta, 2019).

Por lo tanto, en la cooperación educativa internacional, las instituciones de educación superior están obteniendo cada vez más reconocimiento a lo largo de los últimos años como instituciones clave. Desde que se ha admitido el papel de la educación superior en el desarrollo social y económico de los países socios, las universidades públicas juegan un papel legítimo, único y relevante. Las universidades cuentan con una reconocida trayectoria de actuaciones tanto en el ámbito de la acción individual de su profesorado, alumnado y personal de administración y servicios, como de la propia institución universitaria. En ella numerosas personas participan habitualmente y de forma muy dinámica, en todo tipo de actividades para la erradicación de la pobreza y la promoción de un mayor bienestar social, con una especial atención a los más desfavorecidos y a la protección del medioambiente (Fernández, 2015). Hoy día nadie pone en duda la importancia de la cooperación interuniversitaria internacional a la hora de fomentar el desarrollo sostenible de los países. 
Esto ha hecho posible que actualmente la Cooperación Universitaria al Desarrollo pueda definirse, como se estipuló desde la Conferencia de Rectores de la Universidades Españolas (CRUE): ‘Estrategia de Cooperación Universitaria al Desarrollo’ del año 2000:

«como la cooperación de las universidades en los procesos de desarrollo socioeconómico de otros países, que puede llevarse a cabo tanto exclusivamente entre universidades como en colaboración con otros agentes públicos o privados, al objeto de inducir, fomentar o apoyar estrategias de desarrollo» (CEURI-CRUE, 2000, p. 2).

Los principios de la cooperación universitaria al desarrollo se concretan fundamentalmente en la continuidad de las actuaciones, en la complementariedad de las iniciativas, en la reciprocidad y en la solidaridad (CEURI-CRUE, 2000, p. 5.).

Por lo tanto, cualquier acción, política o programa de cooperación siempre deberá instaurarse en el marco de la adopción de relaciones de confianza y en el aprovechamiento de recursos institucionales, que permitan crear líneas y programas de cooperación interuniversitaria, con organismos gubernamentales, no gubernamentales e instituciones educativas, culturales y sociales de diversas características y localizaciones geográficas, en el ámbito nacional e internacional. Estos proyectos tendrán el propósito de establecer diversos convenios y canalizar recursos para programas de asesoría, dotación, capacitación, investigación y desarrollo, pasantías, becas o intercambios culturales y artísticos (López, 2016).

Este tipo de cooperación ha sido impulsada desde la «Declaración Mundial de la UNESCO sobre la Educación Superior en el Siglo XXI: Visión y acción» (Rodrigues, 1998) donde en palabras de Rodrigues (1998) se indica, entre otras cosas, que las organizaciones intergubernamentales y no gubernamentales, los Estados mediante sus programas de cooperación bilateral y multilateral y la comunidad universitaria, deberán promover la movilidad universitaria internacional como medio de hacer progresar el saber y compartirlo, a fin de instaurar y fomentar la solidaridad, como principal elemento de la futura sociedad mundial del saber, por medio de una acción cooperativa a gran escala, con particular acento en la cooperación Sur-Sur.

A raíz de esta Declaración, las universidades de gran parte del mundo, a través de la formación, la educación y la investigación, han implementado estrategias de cooperación al desarrollo convencidas de que deben asumir un papel protagonista en los procesos de desarrollo. No ajenas a esta iniciativa, la Universidad de La Laguna y la Universidad de Cabo Verde han puesto en marcha diversas acciones encaminadas a contribuir a estos propósitos.

La cooperación interuniversitaria en el contexto de la globalización trasciende cada vez más las barreras institucionales, las fronteras nacionales y los límites de los campus universitarios por la emergente economía del conocimiento y el desarrollo extraordinario de las tecnologías de la información y la comunicación. Se organizan redes de aprendizaje y redes de cooperación, constituyéndose verdaderas universidades mundiales que acogen proyectos conjuntos de cooperación basados en la movilidad del profesorado y los estudiantes en función de experiencias formativas; el uso de las tecnologías de la información y la comunicación; la colaboración institucional universitaria; el desarrollo de estudios e investigaciones; Posgrados; proyectos de investigación científicos y tecnológicos, etc. (PIMA, 2006; citado en Madarro, 2011). 
Ahora bien, hay muchas maneras de poner en marcha la cooperación interuniversitaria internacional. En líneas generales, podemos establecer dos grandes perspectivas que nos ayudarán a analizar e interpretar la información recopilada en este estudio: la verticalidad y la horizontalidad.

El modelo de cooperación horizontal se caracteriza porque son los países en desarrollo, en función de sus características, necesidades y posibilidades los que establecen los objetivos de desarrollo en general y de los proyectos de cooperación internacional en particular.

Se produce así una ampliación y diversificación de los objetivos. La variedad de métodos y modalidades de cooperación bajo esta perspectiva pueden alcanzar dimensiones ilimitadas, dando cabida a un espíritu innovador y creativo. Esto también contribuye a una mayor flexibilidad de los programas y proyectos de cooperación y sus procedimientos administrativos; de forma que los objetivos se pueden redefinir, los presupuestos revisar y las acciones rediseñar (González, 2003).

La formulación, el seguimiento y la evaluación de los proyectos se realiza de forma endógena por los países en desarrollo; dando preferencia a la utilización de los servicios de especialistas nacionales. Ello posibilita la elaboración de proyectos más adecuados a cada situación; y permite realizar evaluaciones más acertadas en torno al fracaso o el éxito de las acciones realizadas.

Sin embargo, el modelo de cooperación vertical que lamentablemente existe en la actualidad y que las personas implicadas en este estudio denuncian por su persistencia, se caracteriza porque los objetivos son establecidos por determinados sectores de la cooperación de los países donantes, teniendo como meta final seguir la misma senda que los países desarrollados.

En lo que respecta a los procedimientos administrativos, estos destacan por su rigidez, existiendo escaso margen para innovar y adaptar los diseños de los proyectos a las necesidades y circunstancias tan cambiantes de los países empobrecidos. Las evaluaciones en torno a los resultados de la cooperación son realizadas por determinados expertos internacionales nacidos y/o formados en los países donantes. El desconocimiento de la compleja realidad de los países del Sur y de las causas del subdesarrollo facilita que en numerosas ocasiones, se encuentren las razones de los fracasos de los proyectos en los países receptores, sin llegar a plantearse que los planes elaborados y diseñados pudieran ser incorrectos o inadecuados.

La existencia de condicionalidad en las ayudas ofrecidas por los países donantes es una característica eternamente presente. Detrás de toda ayuda está la idea implícita de la voluntad del «donante». Es decir, «no existe obligación alguna para que esta ayuda se proporcione, estando los receptores a merced de los países desarrollados, a merced de lo que estos deciden: cooperar o no y bajo qué condiciones» (González, 2003, p. 58).

Trataremos de vislumbrar a través de esta investigación en qué medida coexisten actualmente estas dos grandes perspectivas en la cooperación interuniversitaria al desarrollo entre la ULL y la UniCV.

Estas relaciones se iniciaron desde la fundación de la Universidad de Cabo Verde en 2006 y han estado presentes a pesar de los cambios en los equipos de gobierno y los diferentes estamentos creados para su gestión. El periodo concreto de análisis se fundamenta en la creación en 2015 de la Dirección de Cooperación Internacional al Desarrollo en la ULL hasta su cierre en mayo de 2019; acotándose a un periodo de estabilidad en las políticas de externalización implementadas desde dicha universidad. 


\section{Metodología}

Esta investigación se desarrolla en el campo de la Educación Comparada ya que su objetivo es determinar y analizar las diferencias y semejanzas entre la Universidad de Cabo Verde y la Universidad de La Laguna en materia de cooperación interuniversitaria, con objeto de identificar posibles mejoras al reto de la internacionalización de las universidades. Es por tanto un estudio de carácter internacional, acotado al ámbito de la educación superior.

La técnica de investigación más destacada es el análisis de contenido de entrevistas en profundidad, teniendo un carácter cualitativo. La investigación cualitativa está orientada al estudio en profundidad de la compleja realidad social, por lo cual, en el proceso de recolección de datos, la persona que investiga va acumulando numerosos textos provenientes de diferentes fuentes. Según Goetz y Le Compte (1984), el análisis de esta información debe ser abordado de forma sistemática, orientado a generar constructos o categorías y establecer relaciones entre ellos, constituyéndose esta metodología en un camino para llegar de modo coherente a la teorización.

\subsection{Participantes}

Los participantes en este estudio fueron un total de 18 informantes clave, protagonistas de la cooperación interuniversitaria para el desarrollo realizada desde el año 2015 hasta 2019 entre la Universidad de La Laguna y la Universidad de Cabo Verde (ver Tabla 1).

Como puede observarse del total de la muestra: la mitad pertenecen a la Universidad de Cabo Verde, la mitad son mujeres y también la mitad tienen entre 40 y 55 años. El $66,6 \%$ son doctores/as; el 27,9 \% son másteres y tan solo el 5,5 \% goza únicamente de una licenciatura.

A la hora de afrontar el análisis de los datos, se observó la importancia de organizar la muestra en torno a la diferente vinculación con la universidad: 'docencia-investigación' (72,2 \%) y 'gestión-administración-servicios' (27,8 \%). 
Tabla 1.

Características de la muestra -datos generales-

\begin{tabular}{|c|c|c|c|c|}
\hline VARIABLE & $\begin{array}{l}\text { UNIVERSIDAD DE } \\
\text { CABO VERDE }\end{array}$ & $\begin{array}{l}\text { UNIVERSIDAD DE } \\
\text { LA LAGUNA }\end{array}$ & TOTALES & $\%$ \\
\hline \multicolumn{5}{|l|}{ EDAD } \\
\hline (menos de 40 años) & 5 & 0 & 5 & 27,8 \\
\hline $\begin{array}{l}\text { (entre cuarenta y } 55 \\
\text { años) }\end{array}$ & 3 & 6 & 9 & 50 \\
\hline (más de 55 años) & 1 & 3 & 4 & 22,2 \\
\hline \multicolumn{5}{|l|}{ SEXO } \\
\hline Mujer & 5 & 4 & 9 & 50 \\
\hline Hombre & 4 & 5 & 9 & 50 \\
\hline \multicolumn{5}{|l|}{ TITULACIÓN } \\
\hline Doctor/a & 6 & 8 & 14 & 66,6 \\
\hline Máster & 3 & & 3 & 27,9 \\
\hline Licenciada/o & & 1 & 1 & 5,5 \\
\hline \multicolumn{5}{|l|}{ VINCULACIÓN } \\
\hline $\begin{array}{l}\text { Personal docente e } \\
\text { investigador }\end{array}$ & 7 & 6 & 13 & 72,2 \\
\hline $\begin{array}{l}\text { Personal de gestión } \\
\text { política y adminis- } \\
\text { tración }\end{array}$ & 2 & 3 & 5 & 27,8 \\
\hline TOTALES & 9 & 9 & 18 & \\
\hline
\end{tabular}

Nota. Elaboración propia

Las características generales de la muestra respecto al tipo de vinculación con las universidades pueden observarse en la Tabla 2. En el ámbito concreto de la docencia-investigación, se destacó por su relevancia la relación con algunas Ramas de Conocimiento: 'Ciencias' (38,5 \%); 'Ciencias Sociales y Jurídicas' Artes y Humanidades (30,75 \%), otorgando especial importancia a la Educación (30,75 \%) debido al tema tratado en el presente estudio.

Respecto al ámbito de gestión-administración-servicios se organizaron los datos en dos variables: 1) gestión referente a cargos políticos (Vicerrectorados relacionados con Relaciones Internacionales (RRII) y Direcciones de cooperación) (60 \%) y 2) administración-servicios referente a la gestión administrativa de los proyectos de cooperación internacional (40 \%). 
Tabla 2.

Características de la muestra - campo y vinculación con la universidad

\begin{tabular}{|c|c|c|c|c|}
\hline VARIABLE & $\begin{array}{l}\text { UNIVERSIDAD } \\
\text { DE CABO VERDE }\end{array}$ & $\begin{array}{c}\text { UNIVERSIDAD } \\
\text { DE LA } \\
\text { LAGUNA }\end{array}$ & TOTALES & $\begin{array}{c}\% \\
\text { respecto } \\
\text { al total } \\
\text { de la } \\
\text { muestra }\end{array}$ \\
\hline \multicolumn{5}{|l|}{$\begin{array}{l}\text { VICULACIÓN DOCENCIA/ } \\
\text { INVESTIGACIÓN }\end{array}$} \\
\hline $\begin{array}{l}\text { Ciencias Sociales y Jurídicas - } \\
\text { Artes y Humanidades }\end{array}$ & 2 & 2 & 4 & 22,2 \\
\hline $\begin{array}{l}\text { Ciencias Sociales y Jurídicas } \\
\text {-Educación- }\end{array}$ & 2 & 2 & 4 & 22,2 \\
\hline Ciencias & 3 & 2 & 5 & 27,8 \\
\hline SUBTOTALES & 7 & 6 & 13 & 72,2 \\
\hline \multicolumn{5}{|l|}{$\begin{array}{l}\text { VINCULACIÓN GESTIÓN/ } \\
\text { ADMINISTRACIÓN }\end{array}$} \\
\hline Vicerrectorados y Direcciones & 1 & 2 & 3 & 16,7 \\
\hline Gestores y administradores & 1 & 1 & 2 & 11,1 \\
\hline SUBTOTALES & 2 & 3 & 5 & 27,8 \\
\hline TOTALES & 9 & 9 & 18 & 100 \\
\hline
\end{tabular}

Nota. Elaboración propia

\subsection{Instrumentos}

La entrevista estructurada fue el instrumento seleccionado para conocer la perspectiva de los agentes de cooperación interuniversitaria implicados en las relaciones internacionales y proyectos entre la ULL y la UniCV durante cuatro cursos académicos. Se organizó en los ítems indicados en la Tabla 3, permitiendo profundizar en las diversas dimensiones y categorías establecidas: 
Tabla 3.

Dimensiones y categorías establecidas para el análisis de cooperación interuniversitaria ULL-UniCV

\begin{tabular}{ll}
\hline DIMENSIONES & CATEGORÍAS \\
\hline \multirow{2}{*}{$\begin{array}{l}\text { Conceptos clave en torno a la } \\
\text { cooperación }\end{array}$} & Cooperación internacional \\
\cline { 2 - 2 } & Cooperación educativa internacional \\
\cline { 2 - 2 } & Cooperación interuniversitaria la desarrollo \\
\hline Valoración de la cooperación & Modalidades \\
\cline { 2 - 2 } & Acciones \\
\hline & Cooperación internacional \\
\cline { 2 - 2 } & Cooperación educativa internacional \\
\cline { 2 - 2 } & Proyectos \\
\hline Prospectiva interuniversitaria al desarrollo \\
\hline
\end{tabular}

Nota. Elaboración propia

\subsection{Procedimiento}

Las entrevistas se realizaron de manera presencial, mediante Skype, por teléfono y utilizando tecnología multimedia (en este caso WhatsApp) y excepcionalmente (solo 2 entrevistas) se realizaron por correo electrónico.

Las entrevistas a los informantes clave de la ULL fueron realizadas en Tenerife durante el periodo comprendido entre diciembre del 2018 y mayo del 2020.

En Cabo Verde se realizaron en dos fases diferentes. Durante el periodo comprendido entre noviembre del 2018 y marzo del 2019 gracias a una estancia internacional de investigación financiada por la Universidad de La Laguna. El número de entrevistas grabadas ascendió a un total de 6 y pudieron ser procesadas en 2019. Posteriormente, durante una segunda visita realizada en noviembre de 2019, se cumplimentaron varias entrevistas con el objeto de mejorar la muestra del estudio, siendo trascritas entre noviembre 2019 y enero 2020.

\section{Resultados}

A continuación, se presentan los datos obtenidos mediante el análisis comparativo de las dimensiones y categoría establecidas para la recopilación de datos.

Dado que los resultados más relevantes guardan relación con las universidades estudiadas y el tipo de vinculación que las personas entrevistadas tienen con ellas, en este trabajo se analizarán en profundidad estas variables. 


\subsection{Concepciones sobre la cooperación internacional y la cooperación interuniversitaria al desarrollo}

A partir de las entrevistas realizadas, los informantes clave dieron respuesta a las cuestiones que se exponen a continuación.

\subsection{1. ¿A quién beneficia la cooperación internacional?}

Las respuestas ofrecidas en torno a esta relevante cuestión pusieron de manifiesto que se encuentran relacionadas fundamentalmente con la universidad a la que se pertenece y en menor medida con el tipo de vinculación que se tiene con ella.

En líneas generales, los informantes caboverdianos (77,8 \%) manifestaron una concepción de la cooperación de carácter horizontal al relacionar directamente la cooperación internacional con la cooperación entre universidades e instituciones educativas para el desarrollo de ambas partes (A1, A5, A6, A7 y A8, 2018) (A3, A9 y B5, 2019). Como se puede observar, 7 de los 9 agentes de la UniCV coincidieron con la perspectiva siguiente:

«(...) una relación formal entre dos instituciones que es materializada a través de, en nuestro caso, investigadores (...) docentes, estudiantes (...) a través de proyectos y otras colaboraciones y pueden desembocar también en publicaciones, convenios y utilizan un desarrollo, en los dos sentidos, con beneficios para los dos países o las dos entidades que forman parte de ese proyecto conjunto» (A1, 2018).

Las concepciones verticalistas se visualizaron en la sección de Ciencias Sociales y Jurídicas $^{1}$ de la UniCV (A2 y A4, 2018). Este hecho sorprende, dada la alta dependencia en materia de tecnología de la UniCV respecto a universidades extranjeras, cuestión que podía fomentar una visión vertical en el campo de las Ciencias; sin embargo, no es así.

La cooperación vertical prima en la ULL, al poner de manifiesto 6 de las 9 personas entrevistadas $(66,7 \%)$ que la cooperación internacional beneficia fundamentalmente a los países en desarrollo (B2, B3, B4, B6, 2019) (B7 y B8, 2020).

Por ejemplo, desde la sección de Ciencias de la ULL se entiende como el "empleo de fondos para fomentar el desarrollo en países en vías de desarrollo" (B8, 2020); coincidiendo con los planteamientos existentes en la sección de Vicerrectorados y Dirección de la ULL (B4, 2019).

La presencia mayoritaria de una concepción vertical de la cooperación es frecuente en lugares que presentan un mayor nivel de desarrollo económico que otros, como es el caso de Canarias y Cabo Verde. Sin embargo, en Canarias persisten importantes bolsas de pobreza (Fernández, 2019) y problemas comunes con otros territorios fragmentados. No en vano, la Agenda 2030 contempla la relevancia de estas zonas del planeta dada sus desventajas en el ámbito internacional, y la concreta en la Resolución aprobada por la Asamblea General el 14 de noviembre de 2014, Modalidades de Acción Acelerada para los Pequeños Estados Insulares en Desarrollo, conocida como Trayectoria de Samoa (Naciones Unidas, 2014). Estas características e intereses comunes deberían promover una cooperación horizontal que, sin duda, posibilitaría hallar soluciones a problemas comunes que afectan a ambas realidades.

Por ello, a pesar de su escasez y dada la importancia de su fomento en todo el ámbito universitario canario, finalmente se indica que las perspectivas de la cooperación horizontal se hallaron en las Ciencias Sociales y Jurídicas de la ULL (B1 y B5, 2019; B9, 2020).

1 Cuando se indica sección de Ciencias Sociales y Jurídicas en general, se está haciendo referencia tanto a Artes y Humanidades como a Educación. 


\subsection{2. ¿A quién debería beneficiar la cooperación interuniversitaria al desarrollo?}

Acotando el ámbito de análisis a la cooperación entre universidades, las concepciones no se alejan de los planteamientos generales anteriormente expuestos, observándose una mayor exigencia en el deber o compromiso de las universidades a la hora de contribuir a la mejora del mundo, sus sociedades o de las propias universidades.

En función del tipo de vinculación de los informantes clave con sus universidades, se pusieron de manifiesto diferencias a la hora de establecer la finalidad última de la cooperación interuniversitaria. Desde las concepciones más amplias a las más acotadas, se destacan las siguientes:

- La mejora de la educación superior impacta en el desarrollo de las sociedades de los países participantes. Esta ha sido la postura más generalizada al coincidir ocho entrevistados ( 5 de la ULL y 3 de la UniCV), lo que supone un 44,4 \% de la muestra. Sin embargo, varía la pertenencia de las personas entrevistadas a las secciones de cada universidad. En la ULL plantean esta visión desde Educación (B3, 2019 y B9, 2020), Arte y Humanidades (B1, B5, 2019) y Vicerrectorados y Direcciones (B6, 2019). Mientras que, en la UniCV, la ofrecen desde Ciencias (A2, A7, 2018) y Educación (A3, 2019). Es decir, que ambas universidades coinciden al proponer desde Educación que la cooperación en educación superior beneficia el desarrollo de todas las sociedades participantes; y en que, en ninguna de ellas, el sector de Administración planteó esta visión. Así lo expresan algunos de ellos:

«(...) hay muchas iniciativas desde la universidad, la universidad es una institución con vocación universalista (...) Por lo tanto, abierta a la colaboración y al intercambio. De hecho, la investigación en la universidad se concibe cada vez más como un escenario de intercambio de muchos grupos, no solo a nivel nacional sino internacional también ¿Por qué? Porque los problemas que se estudian son problemas internacionales, porque las preocupaciones que hay son preocupaciones internacionales, porque los problemas del conocimiento son problemas del mundo y no son de una universidad, o de un país, o de un grupo determinado. Y eso yo creo que la academia lo entiende bastante bien (...) Otra cosa es que en el alcance de ese intercambio y de esa cooperación, o de esa colaboración, realmente impulse luego, o implique también a otras organizaciones extrauniversitarias, o no universitarias, (...)» (B3, 2019).

«(...) los actores son universidades y la finalidad última del proceso es promover (como su propio nombre indica) un desarrollo educativo global que redunde en un desarrollo social equitativo y sostenible. En este caso las actuaciones son de diferente índole y pueden enfocarse en diferentes aspectos o funciones del sistema, pero la condición última no es buscar el progreso educativo por el progreso, sino el impacto en los niveles de desarrollo sociales (siempre pensando en el concepto de desarrollo humano y sostenible)» (B9, 2020).

- La mejora la educación superior impacta en el desarrollo de las sociedades y las comunidades de los países en vías de desarrollo. Esta visión la plantea únicamente el 16,7 \% de los agentes, dos de la Administración de ambas universidades (A5, 2019 y B7, 2020) y uno de Ciencias de la ULL (B2, 2019): 
«Los países en vías de desarrollo necesitan la formación de doctores, la formación de especialistas, la realización de trabajos con la resolución de problemas de los países y eso es lo que debería de institucionalizarse (...) porque además yo creo que es la única salida que tienen esos países. Aumentar el nivel del conocimiento no solo en primaria, que por supuesto, sino a nivel universitario y de especialistas en todos los campos en desarrollo regional, en salud (que es donde nosotros intervenimos), en política de infraestructura civiles, etcétera. Es importantísimo que tengan personal altamente cualificado $y$, por eso, la línea nuestra de trabajo va en el Posgrado» (B2, 2019).

- Otras concepciones apuntan que la cooperación interuniversitaria fomenta el desarrollo de las universidades, alejándose de la amplitud de la anterior conceptualización. Cuatro de los agentes que pusieron de manifiesto esta visión pertenecen a la UniCV (A4, A6 y A8, 2018 y A9, 2020) y uno solo a la ULL (B4, 2019), lo que supone un 27,8 \% del total de la muestra; no pudiéndose establecer relación alguna con las secciones de pertenencia. Eso sí, esta concepción es compartida por ambos Vicerrectorado/Direcciones:

«La cooperación interuniversitaria para el desarrollo (...) es esencialmente formar para el entendimiento, formar (...) investigadores e investigadoras en el sentido de hacer avanzar los proyectos de la universidad, de crear esa posibilidad de movilidad de conocernos también y (...) para avanzar en el proceso de construcción de conocimiento» $(\mathrm{A} 6,2018)$

Esta limitada concepción sorprende, especialmente en el caso de los Vicerrectorados/ Direcciones, dada la estrecha relación que la cooperación interuniversitaria guarda con los Objetivos de Desarrollo Sostenible (ODS) (2015) y las exigencias de los organismos financiadores que cada vez demandan en mayor medida la transferencia del conocimiento a la sociedad civil.

- La concepción más acotada la encontramos en aquellas manifestaciones que relacionan la cooperación interuniversitaria con el desarrollo de la educación superior exclusivamente en los países en desarrollo. A la hora de identificar los beneficios de la cooperación internacional y la cooperación interuniversitaria, se hallan discrepancias significativas en función de las universidades. Desde la ULL se prioriza la formación desde el sector de Ciencias (B8, 2020); mientras que en la UniCV desde Artes y Humanidades se destaca la investigación (A1, 2018):

«(...) se produce cuando las universidades desarrollan colaboraciones en diversas actividades como investigaciones, publicaciones, encuentros para hacer conferencias, workshops, etc. Existe un diálogo entre investigadores y sobre todo también la internacionalización de esas universidades (...). Son colaboraciones que nos permiten a nosotros (...) el desarrollo de recursos humanos, de todo; el desarrollo de un país a través de las investigaciones (...)» $(\mathrm{A} 1,2018)$.

«Es el empleo de fondos para la mejora de la educación superior en países en desarrollo mediante formación de docentes, formación de estudiantes, intercambio de materiales (...) La formación de expertos y profesionales, sin duda, ayuda al desarrollo de los países en todas las áreas» $(\mathrm{B} 8,2020)$. 
La idea de que la cooperación interuniversitaria impacta en el desarrollo de ambas sociedades y en la mejora de la educación superior de ambos países parece imperar (salvo en el sector de Administración); siendo la primera de esta perspectiva defendida fundamentalmente desde el sector de Educación y la segunda desde los Vicerrectorados y Direcciones de Cooperación de ambas universidades.

\subsection{3. ¿Es posible el beneficio mutuo en la cooperación internacional?}

Los aspectos ético-políticos de la cooperación internacional fueron destacados en varias entrevistas; así como el enfrentamiento entre estos y la práctica ligada a intereses en muchas ocasiones no explícitos que facilitan los procesos de dominación cultural, económica, política... Por citar solo un ejemplo, indicaremos el Proyecto de Competitividad y Desarrollo Empresarial (CEDP) del Banco Mundial realizado con los Países Africanos de Lengua Oficial Portuguesa (PALOP) que ha fomentado la creación de empresas en el sector del transporte que no han prosperado entre Cabo Verde, Guinea Bissau, Guinea Conakry, Angola, Brasil... (A4, 2018).

Los argumentos esgrimidos desde los años 70 desde la Teoría de la Dependencia denunciando el uso de la cooperación como instrumento de dominación del neocolonialismo (Todaro, 1985, 1988) persisten actualmente. Las denuncias y propuestas formuladas frente a la cooperación vertical siguen gozando de enorme relevancia para avanzar hacia una cooperación horizontal (González, 2005).

Ante esta realidad, con el objeto de hacer posible el beneficio mutuo, dos personas en cada una de las universidades de la sección de Ciencias Sociales y Jurídicas, destacaron la importancia de la participación de todos los implicados en la elaboración de las agendas de desarrollo y en establecer relaciones en igualdad que impidan los procesos de dominación (A4 y A6, 2018) (B1 y B3, 2019). Algunas de sus palabras fueron las siguientes:

«(...) en ese proceso cooperativo debemos estar atentos a un conjunto de personas que muchas veces vienen con agendas de fuera en cierta forma impuestas (...) En nuestra universidad, en nuestro país (...) muchas veces tienden a recibir a estas personas de una forma acrítica (...) Muchas veces acabamos por hacer lo que aquí no es prioritario para la universidad o para Cabo Verde (...) ¿Por qué? Porque no tenemos recursos financieros (...) para crear nuestros propios proyectos de acuerdo con nuestras prioridades (...) y nosotros acabamos por aceptar muchas veces proyectos que van en contra de lo que nosotros pensamos que urge (...) Existe una imposición de agendas externas sobre nuestras agendas internas (...)» $(\mathrm{A6}, 2018)$.

«Una cooperación al desarrollo significa una colaboración para cambiar los modos de producción, los modos de pensamiento, los modos de ver el mundo. Y de hacer el mundo para mejorarlo, para cambiarlo, para transformar una realidad determinada, allí o aquí, donde sea. Eso significa que se abre un proceso de cambio profundo, a través de la cooperación para el desarrollo. Y claro, ahí hay una frontera muy fina y muy delicada (...) que es el tema de la dominación. Y ahí es donde entra el factor político: con qué visión y desde qué perspectiva se hace el desarrollo. Con la visión de los que necesitan el desarrollo o con la visión de los que dan el desarrollo ¿Quién se desarrolla? El que recibe o el que da. Si no hay una relación equidistante, entre una y otra, lo más probable es que se produzca una relación de dominación. Y, por lo tanto, una relación de dominio cultural y político del proceso de desarrollo (B3, 2019). 
En la Universidad de Cabo Verde, desde todas sus secciones, salvo la de Vicerrectorado/ Dirección (A1, A2 y A4, 2018) (A5, 2019), se aportaron reflexiones sobre la complejidad de los procesos de cooperación, estableciendo diferencias entre la cooperación Norte-Sur y Sur-Sur:

«Ahora bien, cuando los dos países tienen un nivel de desarrollo igual, como en los casos de los países del tercer mundo (...) se crea el gran problema de cómo la cooperación puede ser válida para el desarrollo de ambos. Ese es un gran problema que tenemos en África (...) hay un problema de la cooperación para el desarrollo entre los países con un mismo nivel de desarrollo, y hay otro gran problema con países de niveles de desarrollo diferente para que uno no domine al otro» $\left(\mathrm{A}_{4}, 2018\right)$.

Haciendo referencia a los nuevos principios de cooperación demandados por los Organismos Internacionales, este mismo informante indica lo siguiente:

«Hay muchas cosas de que hablar (...) con el Fondo Monetario Internacional (FMI) y con el Banco Mundial (BM) (...) Y cuando vemos la historia del desarrollo, últimamente, los países desarrollados, incluso el mismo Banco Mundial, han dicho que se debe cooperar de manera que los países en desarrollo se desarrollen ellos mismos, han creado nuevos protocolos y otras formas de cooperación. Para que no se hiciera la cooperación basada en los intereses del país desarrollado, sino en base a los dos. Para mí es todo un misterio si esto se ha logrado» (A4, 2018).

Sorprende, dado el relevante papel que Cabo Verde puede desempeñar en apoyo a los países de su entorno, que no se haya destacado la importancia de la Cooperación Triangular (OCDE, 2013) como mecanismo de la cooperación internacional para apoyar el desarrollo de África. Y que no se manifieste una visión positiva de la Cooperación SurSur, tras las numerosas experiencias existentes en África que ponen de manifiesto todas sus potencialidades (Kabunda, 2011; Naciones Unidas, 2018; PNUD, 2019).

También debe destacarse el hecho que solo el $44.4 \%$ de los agentes claves se manifestarán a este respecto; siendo comprensible que la mayoría de ellos pertenecieran a la UniCV. Sólo dos personas de la ULL aportaron reflexiones, ambas de la sección de Ciencias Sociales y Jurídicas.

\subsection{4. ¿Existen mecanismos que facilitan la gestión de la cooperación interuniversitaria?}

A este respecto, ocho personas indicaron sus críticas con la forma en que los proyectos son gestionados desde ambas universidades (A2, A4 y B2, 2018; A5, B1, B3, B6, 2019; B9, 2020). Esto supone un 44,4 \% de la muestra y pertenecen mayoritariamente a la ULL. Destacaron la necesidad de principios éticos, de normativas claras, de apoyo institucional, idiomas, flexibilidad, financiación... sugerencias, muchas de ellas, presentes en la Declaración de París sobre la Eficacia de la Ayuda al Desarrollo (2005) (OECD, 2005/2008).

Desde todas las secciones se realizaron propuestas, exceptuando desde los Vicerrectorados/Direcciones de la UniCV y del sector de Administración de la ULL. Entre las necesidades más destacadas, se indicaron: 
a. Una gestión de la cooperación a través de un departamento o institución.

b. Redes de contactos para poner en marcha e implementar proyectos.

c. Una normativa operativa.

d. Una ética del desarrollo.

Las dificultades con el lenguaje y los idiomas también fueron mencionadas:

«(...) la cooperación con países africanos tiene un problema importante (...) y es el idioma (...) Nuestros alumnos, por ejemplo, cuando vienen profesores de allá, a veces no van a la conferencia (...) porque no hay traducción simultánea (...) Tenemos, por ejemplo, becas del Gobierno de Canarias para ir a (...) a países de habla francesa (...) y nadie las pide porque no tienen ningún nivel de francés (...) Nuestros propios estudios no están adaptados a una realidad que tenemos al lado (...) No tenemos desarrollado másteres que sean por red en otros idiomas, nuestro profesorado, por ejemplo, no está formado (...) Es patente de que si España quiere tener mayor relevancia en esos contextos tiene que abrir Institutos y Centros de idiomas (...) Hay alumnado que está loco por venirse para acá, porque yo voy allá y me preguntan por becas, pero claro, si luego le piden un nivel que no tiene $(. .) ».(\mathrm{~B} 1,2019)$

Otra aportación fue la realizada por informantes clave de ambas universidades, especialmente por la ULL (3 de los 4 informantes) al enfatizar la falta de flexibilidad con respecto a la participación de agentes externos a la universidad. Este hecho es muy comprensible dado que normalmente los responsables y encargados de la gestión y justificación de los proyectos de cooperación interuniversitaria son miembros de la ULL.

Los protocolos que existen para gestionar la colaboración entre instituciones universitarias y no universitarias dificultan en gran medida este tipo de intercambios y también los son a la hora de propiciar la movilidad del profesorado; crítica realizada desde la sección de Educación de la UniCV y de la ULL (A4, 2018; B9, 2020; B3, 2019) y desde Artes y Humanidades de la Universidad de la Laguna (B1, 2019).

«Creo que saliéndonos del discurso de donantes y beneficiarios que en muchas ocasiones impregna este campo, cualquier cooperación entre universidades puede generar sinergias muy interesantes. Ahora bien, eso implicaría trascender los modelos de proyectos al uso que son muy rígidos $\mathrm{y}$ en los que muchas veces, las universidades cooperan como un ente aislado del contexto social donde se encuentran, o enfatizan la promoción de sus investigadores en una determinada área» (B9, 2020).

«(...) las universidades tienen un sistema de regulación laboral, es decir, de sus categorías contractuales, que combina mal la movilidad con la estabilidad (...) Los protocolos a través de los cuales se realiza ese intercambio son diferentes en cada país, en cada universidad (...) (B3, 2019).

La crítica respecto a los escasos fondos destinados a la cooperación interuniversitaria no podía faltar; sin embargo, sorprende que fuera minoritaria y solo se indicara en la ULL (tan solo 4 personas) esta cuestión como relevante (B1, B2, B4 y B6 2019). El hecho de que ni el Vicerrectorado/Dirección de la UniCV, ni la sección de Administración de la ULL hayan hecho sugerencias para la mejora de la gestión, sugieren la necesidad de 
establecer mejores conexiones entre todas las secciones en cada universidad. Ello posibilitaría un enriquecimiento mutuo y la puesta en prácticas de mejoras sugeridas.

\subsection{Valoraciones de la cooperación interuniversitaria entre la UniCV y la ULL}

A partir de las entrevistas realizadas, los informantes clave dieron respuesta fundamentalmente a dos cuestiones: 1) Relevancia de la cooperación interuniversitaria entre Cabo Verde y Canarias; y 2) Sugerencias para su mejora.

\subsection{1. ¿Es relevante la cooperación interuniversitaria entre Cabo Verde y Canarias?}

Desde todas las secciones de ambas universidades se manifiesta la importancia de esta cooperación. Un total de 11 agentes (A1, A5 y A7, 2018) (A3, B2, B3, B4, B5, B6 y B9, 2019) (B7 y B8, 2000) coinciden en su valía, lo que supone el 61,1 \% de la muestra:

«Lo más relevante es la movilización de profesorado y alumnado, es una experiencia muy enriquecedora. En términos de aprendizaje es una gran contribución al conocimiento y aumenta la perspectiva global. Es una experiencia muy provechosa desde el punto de vista de las publicaciones, los cursos, el análisis de los sistemas educativos europeos y africanos» (A3, 2019).

También la definen como gratificante, además de fructífera; destacando los aspectos comunes a ambos territorios como una de las fortalezas de la cooperación UniCV/ULL:

«La condición compartida de universidades insulares y ultraperiféricas nos da una problemática común en diversos ámbitos. Por otra parte, Canarias puede servir como ejemplo o, en muchos casos, como contraejemplo, de desarrollo para Cabo Verde. Y creo que debería ser un aspecto prioritario de la cooperación fomentar el desarrollo sostenible de ese país» $(B 7,2020)$.

Los otros siete entrevistados, es decir el 38,9 \% de la muestra, sin obviar beneficios, destacan fundamentalmente los errores que la caracteriza: la primacía de los intereses comerciales canarios, la intromisión de instituciones no universitarias, el exceso de burocratización y la falta de proyectos globales al desarrollo a largo plazo. Es relevante que la mayoría de estas opiniones surgen desde la UniCV (A2, A4, A6 y A8, 2018) (A5, A9 y B1, 2019). Respecto a los sectores de pertenencia hay que indicar que todos los sectores de UniCV; y ninguno, salvo un informante de la ULL perteneciente a Artes y Humanidades, denuncian los errores de la colaboración que se analiza en este estudio.

Se observan más coincidencias entre las personas entrevistadas con respecto a los límites de los países terceros como Cabo Verde, que no pueden ser cabeza de lista en la gestión de los proyectos, formando parte como participante o invitado. Esta circunstancia debería de ser revisada, especialmente considerando que desde 2008 Cabo Verde es un país de desarrollo medio ${ }^{2}$. Si ya no recibe tantos fondos como receptor, bien podría considerarse gestor.

2 Cabo Verde salió del grupo de los países menos adelantados PMA en diciembre de 2007 (Naciones Unidas, 2008, pág. 13). 
«Lo importante sería que Cabo Verde pudiera coordinar los proyectos porque al participar como país tercero, ni recibe financiación ni tiene capacidad de decisión (...) Lo que hace falta es que Cabo Verde pueda liderar un proyecto, no participar únicamente como socio. Tienen muchas limitaciones en sus responsabilidades. Sin embargo, en los Erasmus +, Cabo Verde sí recibe los fondos y es responsable de su gestión» (A9, 2019).

«Nuestros profesores, la mayoría, tienen la experiencia europea o americana, estudiaron fuera de Cabo Verde a nivel de doctorado, por ejemplo, o máster (...) Son personas que tienen una buena formación académica y pueden también ayudar al desarrollo y la cooperación» (A8, 2018).

Frente a esta realidad, un hecho que destaca la ULL es la elevada preparación de la UniCV para gestionar los proyectos de cooperación internacional:

«(...) la cooperación con Cabo Verde funciona bien porque la estructura universitaria allí es una estructura muy europea (...) preparada para responder rápido a las demandas burocráticas de Europa. Por lo tanto, Cabo Verde está preparada para interaccionar con nosotros, de forma dinámica y compartimos los mismos ámbitos de trabajo, de investigación (...)» $(\mathrm{B} 6,2019)$.

\subsection{2. ¿Puede mejorarse la cooperación interuniversitaria entre Cabo Verde y Canarias?}

Ante las dificultades anteriormente señaladas, las propuestas realizadas desde las dos universidades fueron muy enriquecedoras y pueden contribuir en gran medida al futuro de las relaciones entre ambas y a la cooperación interuniversitaria, en general. Estas han sido agrupadas en los siguientes objetivos, caracterizados por su gran diversidad tanto en función de las universidades como de los sectores de pertenencia. Se presentan por orden de mayor a menor número de demandantes.

\section{- $\quad$ Atender a los intereses de todos los participantes}

Seis informantes reclamaron esta cuestión (el 33,3 \% de la muestra), 5 de ellos pertenecientes a la UniCV y de diferentes secciones, salvo el Vicerrectorado/Dirección. En la ULL, la sección de Artes y Humanidades es la única en proponerla, indispensable si se desea avanzar hacia una cooperación de carácter horizontal (A1, A2 y A8, 2018; A3, A5, B5, 2019). Para que los intereses de todos los participantes puedan ser atendidos, varios informantes reclaman la elaboración de planes y/o agendas compartidas:

«Para mí cualquier tipo de cooperación debe tener un plan. En la planificación deben estar claras las responsabilidades de cada institución, e independientemente de si una de las universidades es más internacional, o si la otra es más antigua. La cooperación debe ser muy compartida, porque tanto de un lado como de otro, las universidades pueden ofrecer a través de sus experiencias (...) Unas pueden ser más desarrolladas y otras menos desarrolladas; más, en el sentido educativo, una posición de jerarquía de poder no es buena ya que la cooperación no es mutua» (A1, 2018). 
Un ejemplo de proyecto caracterizado por este principio de funcionamiento es el titulado Redes de cooperación interuniversitaria Canarias-África: investigación, innovación y evaluación desde metodologías colaborativas en políticas de igualdad (Red CIMPI) impulsado por la Universidad de La Laguna ${ }^{3}$. Sin embargo, desde el sector de Vicerrectorado/Dirección de esta universidad $(B 6,2018)$ se denuncia que los intereses de las propias universidades inciden en escasa medida en la cooperación interuniversitaria, sean desarrolladas o no. Las políticas vienen determinadas desde el exterior:

«La Macaronesia es una mezcla entre el Gobierno de Canarias y los fondos de la Unión Europea (...). Canarias ha señalado a Marruecos, Mauritania, Senegal, Cabo Verde y Guinea, como países receptores de la cooperación, y nosotros como instituciones canarias, tenemos que alinearnos con nuestro gobierno, para unificar esfuerzos (...) La Unión Europea habilita fondos estructurales para diferentes áreas, como el Atlántico Norte, la Región Mediterránea, los Balcanes, Latinoamérica, África, etc. (...) Entonces igual que nosotros miramos al gobierno de Canarias, el gobierno de Canarias mira al gobierno nacional, y el nacional mira al europeo. Aquí todos miramos hacia arriba (...)» $(\mathrm{B} 6,2018)$.

\section{- Transferir conocimientos a las sociedades}

Cinco de los agentes universitarios de la UniCV y de la ULL esgrimieron sólidos argumentos sobre la necesidad de tener en cuenta las demandas sociales de los lugares donde se desarrolla la cooperación, se postula la necesidad de favorecer el desarrollo de las comunidades y el compromiso de las universidades con sus respectivas sociedades. Lo que supone el 27,8 \% de la muestra (A6, 2018; A3, B5, 2019; B8 y B9, 2020). Ni la universidad ni los sectores de pertenencia son factores que determinen esta propuesta. Lo único que puede afirmarse es que desde Vicerrectorado/Dirección y la Administración de ambas universidades no se manifiestan al respecto.

«Es necesario una mayor dimensión de la extensión universitaria. Tenemos que integrarnos con la sociedad y realizar más trabajo de campo en la comunidad. Identificar qué necesitan las comunidades y la sociedad. Debería de existir un Comité de Pilotaje que formara parte del proyecto y velera por esta cuestión, Si no, no veo posibilidades de impacto, de proceso de apropiación, seguimiento y evaluación. En definitiva, de formar parte de los procesos. Veo esfuerzo para las universidades, pero para la sociedad no, sinceramente no» $(\mathrm{A} 3,2019)$.

\section{- $\quad$ Fomentar las titulaciones doctorales caboverdianas}

Cinco de los agentes universitarios entrevistados pertenecientes tanto a la Universidad de Cabo Verde como a la Universidad de La Laguna (A6, A7, A8, 2018; B5, 2019; A9, 2020) coincidieron en afirmar que la cooperación interuniversitaria es fundamental para los procesos de enseñanza y aprendizaje de la UniCV, para fomentar la puesta en marcha de titulaciones propias caboverdianas (27,8 \% de la muestra). No se menciona este mismo beneficio para la ULL, salvo en un caso de la sección de Ciencia de la UniCV $(\mathrm{A} 8,2018)$. Estos son algunos de los comentarios donde se reflejan ambas posturas:

3 Tanto los objetivos como la metodología, las actividades y los resultados del proyecto Red CIMPI pueden ser consultados en: https://www.cimpiull.com/ 
«Se ha creado un buen laboratorio, se ha formado gente, estamos formando a doctores aquí y se están haciendo tesis doctorales aquí sobre problemas de allí, de Cabo Verde, problemas de sanidad de Cabo Verde. Y el paso ulterior es que creen un equipo de investigación en aquella universidad (...) Es muy relevante porque, por ejemplo, necesitan doctores para ser una universidad homologable internacionalmente y debo decir que en Cabo Verde el número de doctores va creciendo gradualmente en la Universidad, pero todavía no tiene el porcentaje (...)» (B2, 2019).

Destaca la sugerencia de una de las personas entrevistada del ámbito de Vicerrectorado y Dirección de la UniCV al señalar la necesidad de desarrollar también programas propios de licenciatura y de postgrado (A9, 2020).

\section{- $\quad$ Formar para la cooperación interuniversitaria al desarrollo}

Se detecta la necesidad de formación en materia de cooperación educativa internacional en los tres sectores universitarios: profesorado, alumnado y administración/servicios; por parte de tres agentes universitarios de la ULL (B1, B3, B2, 2019) y dos agentes universitarios de la UniCV (A3, 2019; A4, 2018) (27,8 \% de la muestra). En ninguna universidad se manifestaron al respecto los entrevistados del Vicerrectorado/Direcciones y Administraciones.

El objetivo de esta formación sería que la cooperación se lleve a cabo de una manera óptima; es decir, que haya motivación y profesionalidad dado que quien no conoce los parámetros de la cooperación no puede sentir, ni conocer los beneficios que se consiguen con la misma.

«Las instituciones no son cosas abstractas, son personas que viven dentro, que hacen, piensan, realizan y hablan de una determinada manera; y al final eso es la clave del asunto. Si las personas quieren la cooperación existe, si las personas no quieren por muchas políticas que queramos poner en la cabeza no va a haber nunca cooperación. Y eso implica una dimensión pedagógica fundamental y es que es la comunicación entre las personas la que posibilita el intercambio, la cooperación. Y eso es una cuestión educativa. El fondo y la raíz de todo proceso de cooperación, es por definición educativa (B3, 2019).

\section{- Dotar de transparencia los objetivos, procesos y resultados de la cooperación}

Solo cuatro de los agentes universitarios entrevistados de la UniCV estimaron necesario mencionar la necesidad de mayor transparencia en la gestión de los proyectos de cooperación (A2, A7, 2018; A5, A9, 2019). Lo hicieron todas las secciones, salvo la de Ciencias Jurídicas y Sociales (el 22.2 \% de la muestra). Los comentarios que se muestran a continuación fueron los aportados por el agente universitario de la UniCV identificado como A2:

«Como investigador de Cabo Verde apuesto por la utilización de los resultados de la investigación para ambas partes (...) Yo tuve acceso a los resultados, pero no tengo potestad para comunicar los resultados. No puedo transferir los resultados a otras personas y, por tanto, no puedo dar continuidad al proyecto. Es necesario que Canarias y Cabo Verde hablen sobre esta cuestión» (A2, 2018). 


\section{- Abrir las fronteras para los actores en cooperación interuniversitaria}

Existen problemas de carácter administrativo que no son generados desde las universidades, pero dificultan en gran medida el proceso de los proyectos de cooperación que estas desarrollan. Aunque los problemas los han sufrido especialmente el profesorado y alumnado caboverdiano, lo cierto es que este asunto es demandado especialmente desde la ULL (A4, B1 y B2, B3, 2019) (el 22.2 \% de la muestra). Todas las secciones de la ULL lo plantean, salvo Vicerrectorado/Dirección y Administración. Y solo desde la sección de Educación en la UniCV. Algunas de las cuestiones planteadas quedan reflejadas en los siguientes comentarios:

«Yo para ir tengo que sacar tales papeles, tales declaraciones, voy a la embajada para mostrarlo y he de esperar (...) vas a encontrarte con problemas para que la cooperación tenga un impacto mayor (...)» (A4, 2018).

«No es tan complicado para que nosotros nos movamos (...) pero, después, cuando viene un estudiante o un doctorando te ponen todos los problemas o no te dan el visado. Porque tienes que pedirlo con tres meses de antelación. Porque les piden montón de papeles, de bancos (...)» (B1, 2019).

\section{- Analizar las necesidades y consecuencias de las iniciativas de cooperación}

Otras reflexiones señalan la necesidad de tener en cuenta las consecuencias de la cooperación, especialmente cuando priman los intereses comerciales y empresariales extranjeros sobre las necesidades reales de la población. Frente a esta situación, la Universidad se describe como un motor para el desarrollo con responsabilidad social. Es la sección de Administración de ambas universidades la que realiza este aporte (A5, 2019; B7, 2020).

«(...) la cooperación entre universidades a veces se usa como excusa entre los países para que también haya crecimiento empresarial extranjero en la zona. Esa es de veras la preocupación, sí. Por lo tanto, es necesario fomentar que las universidades también ayuden a avanzar a las sociedades y que consiga producir conocimiento que pueda ser transferido a la sociedad (...) y tener presente cuales pueden ser los daños si las cosas no se hacen bien» $(\mathrm{A} 5,2019)$.

«Creo que las universidades son un actor importantísimo de la cooperación al desarrollo, especialmente desde el momento en que se generaliza el concepto de responsabilidad social como eje transversal de la actividad universitaria. Creo que es importante mantener los proyectos educativos, tanto los que implican la dotación de becas para alumnos entrantes como los orientados al diagnóstico de las necesidades y el desarrollo de programas de estudios adaptados a las necesidades locales, pero debemos avanzar más en la línea de proyectos que (...) permiten desarrollar actividades de investigación y transferencia de recursos a las instituciones locales de los países perceptores de ayuda. En el caso de las universidades canarias, es obvio que debemos prestar mayor atención a los países africanos más cercanos» $(\mathrm{B} 7,2020)$. 


\section{- Elaborar conjuntamente los proyectos y darles continuidad}

Desde la sección de Vicerrectorados y Dirección de la UniCV y de la ULL (A9, 2019; B4, 2019) se hacen diferentes propuestas en cuanto a la atención de las necesidades locales. Como la necesidad de una financiación previa a los proyectos, que permita que estos no surjan de la imaginación del agente universitario involucrado en el desarrollo del mismo. De hecho, los proyectos exitosos, normalmente han sido elaborados de manera conjunta, en el terreno. Deberían de volver a ponerse en marcha convocatorias como las abiertas por la Agencia Española de Cooperación Internacional al Desarrollo (AECID) hace unos años para la formulación de proyecto de manera horizontal, utilizando en numerosas ocasiones metodologías colaborativas. A este respecto, puede ser consultado el proyecto Redes para la formación y la investigación en Cooperación y Educación para el Desarrollo formulado conjuntamente ente profesorado caboverdiano y canario (González et al, 2016).

«Es necesario que se permita la financiación previa al desarrollo para hacer un análisis de necesidades en cada proyecto. Eso es fundamental, que se permita a los equipos de investigación ir al país antes de emprender el proyecto. Para que puedan hacer un análisis de necesidades in situ, no imaginando (...) Y la consulta a los propios habitantes, a los que están viviendo allí (...) Porque la idea es mantener lo hecho ¿no? (...) Por eso es tan importante el análisis de las necesidades para hacer un proyecto que luego sea sostenible (...) (B4, 2019).

\section{- $\quad$ Otras sugerencias de mejora}

Concretando otros aspectos a mejorar, cuatro personas entrevistadas de ambas universidades realizan más aportaciones (A4, 2018; A3, B1, B3, 2019):

- Reforzar la organización del área de internacionalización de la universidad (B3, 2019).

- $\quad$ Crear un Secretariado específico para África (B3, 2019).

- $\quad$ Abrir un observatorio permanente en sus relaciones con África y otro con Latinoamérica; con la infraestructura necesaria: administrativos, personal, político... que fueran los que dinamizan los proyectos de cooperación (B3, 2019).

- $\quad$ Configurar una red de profesorado que incluya a África para el trabajo conjunto entre sociedades que están próximas, que tienen retos comunes como la inmigración, género, ... «(...) son áreas en la que se podría trabajar conjuntamente, aportando diferentes formas de afrontar estos retos. Hay mucho que reforzar» (A3, B1, 2019).

Priorizar proyectos sobre políticas de Igualdad, codesarrollo y diversidad cultural (B1, 2019).

Promover la movilidad y el intercambio. «Incluso másteres conjuntos entre universidades, que se pueda mover la gente, no. A mí eso me parece que es muy interesante» (B1, 2019).

- $\quad$ Posibilitar el acceso a la biblioteca de la ULL (A4, 2018). 


\section{Conclusiones}

Atendiendo a los datos recopilados se puede concluir que en la UniCV prima una visión horizontal de la cooperación internacional frente a la vertical de la ULL. Sin embargo, si nos sumergimos en el espacio superior de la educación, ambas se acercan al entender que la cooperación interuniversitaria debería de beneficiar a las sociedades de todos los países involucrados. La idea de beneficio mutuo se hace presente, aunque en menos de la mitad de los entrevistados.

Existe una clara preocupación en los informantes caboverdianos respecto a los procesos de dominación asociados a la cooperación internacional. Y más concretamente, sobre los intereses comerciales de Canarias en la zona, denunciando la injerencia de instituciones externas en las prioridades de la UniCV.

Otras limitaciones relevantes hacen referencia al exceso de burocratización, lo cual obliga a destinar excesivo tiempo a la gestión en detrimento del diseño e implementación de actividades de calidad a favor del desarrollo. La bondad de las propuestas también se ve afectada por la carencia de proyectos globales a largo plazo dificulta el logro de objetivos que requieren de tiempo prolongado; esto es especialmente relevante en lo que concierne a la formación y educación. Prima la valoración de los resultados frente a los lentos procesos de diálogo y búsqueda de consenso, cuestiones esenciales para poder cooperar de manera horizontal.

Por otro lado, los límites impuestos a Cabo Verde para poder liderar los proyectos de cooperación interuniversitaria, poco favorecen el desarrollo de su sociedad de manera autónoma. Y eso que es un país que ha demostrado, a lo largo de muchos años, su elevada capacidad para promover iniciativas internacionales de colaboración mediante una destacada actitud proactiva.

A pesar de estos riesgos, la mayoría de los informantes clave opinan que la cooperación interuniversitaria UniCV/ULL es relevante, además de fructífera y gratificante para ambas partes:

«(...) somos archipiélagos que funcionan como puentes entre continentes, tenemos una situación privilegiada, la situación es ideal para (...) apoyar el desarrollo en el continente africano» (B8, 2020).

Pero también consideran que mejoría de manera significativa si se establecieran los siguientes objetivos:

-Atender a los intereses de todos los participantes

- Transferir conocimientos a las sociedades

- Fomentar las titulaciones doctorales caboverdianas

- Formar para la cooperación interuniversitaria al desarrollo

- Dotar de transparencia los objetivos, procesos y resultados de la cooperación

-Abrir las fronteras para los actores en cooperación interuniversitaria

-Analizar las necesidades y consecuencias de las iniciativas de cooperación

-Elaborar conjuntamente los proyectos y darles continuidad. 
Todo lo anteriormente expuesto, nos permite afirmar la persistencia de la verticalidad de la cooperación internacional interuniversitaria, la cual parece que se origina desde cuatro ejes:

Un primer eje proviene de la agenda establecida por los organismos e instituciones a nivel mundial que la diseñan sin tener en cuenta las necesidades y demandas de la mayoría de las personas que se encargan del desarrollo de la cooperación. Tanto de los países que organizan la cooperación, como de los países que la reciben. La realidad es que no existen vías para obtener la visión de la cooperación internacional que tienen la mayoría de los agentes que participan en la misma. Ello produce que, con el uso de la agenda de cooperación internacional establecida por los organismos internacionales, solo se beneficien determinados aspectos de la sociedad y de la comunidad global.

Un segundo eje proviene de las instituciones que envuelven a la universidad como autoridades locales einstituciones públicas, como embajadas, ayuntamientos, el Gobierno de Canarias y el Cabildo de Tenerife en este caso, así como las empresas vinculadas a las mismas. Estas últimas parecen tener el control para acceder a los fondos destinados a la cooperación, especialmente en los últimos años, primando los intereses canarios.

El tercer eje se puede decir que proviene del propio gobierno de las universidades, pues parece que no existe la manera de tener información sobre los patrones de gestión de los fondos destinados a la cooperación internacional. Tampoco parece que haya organización y comunicación dentro de las universidades sobre la cooperación interuniversitaria para atender las necesidades y demandas de los agentes universitarios que participan en la misma. Ni se produce una evaluación de la calidad y efectividad de los proyectos ejecutados.

El cuarto eje se produce cuando la cooperación se proyecta hacia el exterior, en este caso cuando se desarrolla con Cabo Verde. Pues en ella también se reflejan los tres ejes anteriores. Esto se debe a que la cooperación ya está prediseñada y sin posibilidad de modificarse.

Por lo tanto, atendiendo a estos cuatro ejes, se puede afirmar que deben mejorarse los canales de comunicación y los registros de la cooperación internacional que se llevan a cabo entre la Universidad de La Laguna y la Universidad de Cabo Verde. Al no incluirse las necesidades y demandas de la mayoría de los agentes universitarios y sociales que participan y contribuyen en la cooperación internacional, no se produce un desarrollo social y humano en su plenitud.

Urge la necesidad de transparencia y una participación equitativa en cooperación, de tal manera que los países en desarrollo puedan involucrarse de manera igualitaria, con el mismo nivel de participación en el diseño y gestión de la cooperación internacional, produciéndose un impacto real.

Por último, la Universidad también debe responsabilizarse y comprometerse a mejorar la gestión de la cooperación internacional que se desarrolla en la sociedad. La transferencia del conocimiento en pro del desarrollo sostenible y equitativo debe comenzar a ser una prioridad en las universidades públicas. Únicamente de este modo, pueden ser auténticos agentes de Desarrollo Humano Sostenible, abandonando la tradicional cooperación vertical unidireccional que va mayoritariamente desde los organismos internacionales a las autoridades locales e instituciones públicas y, en menor y última instancia, a las universidades y los agentes sociales.

«Creo que las universidades son un actor importantísimo de la cooperación al desarrollo, especialmente desde el momento en que se generaliza el concepto de responsabilidad social como eje transversal de la actividad universitaria» $(\mathrm{B} 8,2020)$. 


\section{Referencias}

CEURI-CRUE. (2000). Estrategia de Cooperación Universitaria al Desarrollo. Córdoba, 3 de marzo, 2000. http://www.ocud.es/files/doc555/escude2000.pdf

Fernández Cuesta, C. (2015). La cooperación universitaria al desarrollo y su proyección futura. Encuentros multidisciplinares, 17, (49), pp. 66-81. http://www.encuentrosmultidisciplinares.org/Revistan\%C2\%BA49/Carmen_Fern\%C3\%A1ndez_Cuesta. pdf

Fernández Maíllo, G. (2019) (coord.): Informe sobre Exclusión y Desarrollo Social en

Canarias. Resultados de la Encuesta sobre Integración y Necesidades Sociales 2018. Informes Territoriales 2019. Fundación FOESSA/ Cáritas Española Editores, Madrid. [Recurso electrónico] Disponible en: https://caritastenerife.org/wpcontent/uploads/2019/o9/Canarias-VIII-Informe-FOESSA-1.pdf

García de la Torre Gómez, M. 2013. Cooperación Educativa Internacional. En González

Pérez, M.I, García de la Torre Gómez, M., \& Rodríguez Spínola, C. (2013). Guía de cooperación educativa internacional y para el desarrollo (pp. 67-83). Biblioteca Nueva Universidad.

García de la Torre Gómez, M., y González Pérez, M.I. (2006). La cooperación educativa entre escuelas. Universidad de La Laguna.

Goetz, J. P. y LeCompte M.D. (1984). Etnografía y diseño cualitativo en investigación

educativa. Ediciones Morata S. A. https://upeldem.files.wordpress.com/2018/o3/libroetnografc3ada-y-disec3b1o-cualitativo-en-investigacic3b3n-educatica-j-p-goetzy-m-d-lecompte.pdf

González Pérez, M.I. (2003). La cooperación educativa internacional ante la rebeldía de las culturas. Una propuesta de pluralidad mestiza para América Latina. [Tesis Doctoral de la Universidad de La Laguna]. https://riull.ull.es/xmlui/bitstream/ handle/915/9952/cs137.pdf?sequence=1\&isAllowed $=y$

González Pérez, M. I. (2005). La cooperación Educativa ante la rebeldía de las culturas. IEPALA.

González Pérez, M.I., et al. (2016). Buscando nuevos caminos para la cooperación interuniversitaria entre Cabo Verde y Canarias. En Valle, J.M. y Núñez, J.A. (Eds.), Educación, supranacionalidad y ciudadanía (pp. 103-116). Fundación Santillana.

Kabunda, M. (2011). África y la Cooperación con el Sur desde el Sur. Ediciones Catara.

López López, V. (2016). Reseña de un caso de cooperación interinstitucional entre Educafin, Injug y el Sabes. Revista Coespe. http://www.revistacoepesgto.mx/ revistacoepes5/index.php/component/content/article/54-diciembre-2012/179resena-de-un-caso-de-cooperacion-interinstitucional-entre-educafin-injug-y-elsabes. 
Madarro, A. (2011). Redes de movilidad académica para la cooperación e integración regional en Iberoamérica. Revista Ibero Americana de Educacion. Septiembrediciembre 2011 (57). https://rieoei.org/historico/documentos/rie57ao3.htm

Naciones Unidas. (2008). Los Países Menos Adelantados. Informe 2008. https://unctad.org/es/system/files/official-document/ldc2008_sp.pdf

Naciones Unidas. (2014). 69/15 Modalidades de Acción Acelerada para los Pequeños Estados Insulares en Desarrollo (Trayectoria de Samoa). https://unctad.org/ system/files/official-document/ares69d15_es.pdf

Naciones Unidas. (2018). Buenas Prácticas en la Cooperación Sur-Sur y Triangular para el Desarrollo Sostenible - Volumen 2. https://www.unsouthsouth. org/2018/11/28/good-practices-in-south-south-and-triangular-cooperation-forsustainable-development-vol-2-2018-in-seven-languages/?lang=es

OCDE. (2005/2008). Declaración de París sobre la eficacia de la ayuda al desarrollo (2005) y Programa de Acción de Accra (2008). https://www.oecd.org/dac/ effectiveness/34580968.pdf

OCDE. (2013). Triangular Co-operation: What's the literature telling us?. https:// www.oecd.org/dac/dac-global-relations/OECD\%20Triangluar\%20Co-operation \%20Literature\%20Review\%20June\%202013.pdf

PNUD. (2019). Soluciones de Desarrollo del Sur para el logro de los Objetivos de Desarrollo Sostenible. El PNUD y la Cooperación Sur-Sur. https://www.ar.undp. org/content/argentina/es/home/library/PNUDGlobal/PNUDCSS.html

Rodrigues Dias, M.A. (1998). Declaración Mundial de la UNESCO sobre la Educación Superior en el Siglo XXI: Visión y acción. Conferencia Mundial sobre la Educación Superior. Educación Superior y Sociedad. 9(2), 3-6. https://www.iesalc.unesco. org/ess/index.php/ess3/issue/view/21/21

Todaro, Michael (1985). Economic Development in the Third World. Longman.

Todaro, Michael (1888). El desarrollo económico del Tercer Mundo. Alianza Editorial.

Villalta, J.M. (2019). La contribución de las universidades al desarrollo económico y social.

Fundación CYD, https://www.fundacioncyd.org/contribucion-de-las-universidades-aldesarrollo/ 